\title{
Spatiotemporal coordination of trophoblast and allantoic Rbpj signaling directs normal placental morphogenesis
}

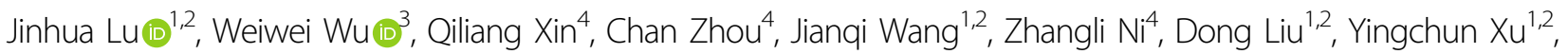 \\ Yongqin Yu ${ }^{1,2}$, Ningjie Yang ${ }^{1,2}$, Yang Sun ${ }^{1,2}$, Bo He $\mathbb{E}^{1,2}$, Shuangbo Kong ${ }^{1,2}$, Shumin Wang ${ }^{5}$, Chao Wang ${ }^{4}$ and \\ Haibin Wang ${ }^{1,2}$
}

\begin{abstract}
The placenta, responsible for the nutrient and gas exchange between the mother and fetus, is pivotal for successful pregnancy. It has been shown that Rbpj, the core transcriptional mediator of Notch signaling pathway, is required for normal placentation in mice. However, it remains largely unclear how Rbpj signaling in different placental compartments coordinates with other important regulators to ensure normal placental morphogenesis. In this study, we found that systemic deletion of Rbpj led to abnormal chorioallantoic morphogenesis and defective trophoblast differentiation in the ectoplacental cone (EPC). Employing mouse models with selective deletion of Rbpj in the allantois versus trophoblast, combining tetraploid aggregation assay, we demonstrated that allantois-expressed Rbpj is essential for chorioallantoic attachment and subsequent invagination of allantoic blood vessels into the chorionic ectoderm. Further studies uncovered that allantoic Rbpj regulates chorioallantoic fusion and morphogenesis via targeting Vcam1 in a Notch-dependent manner. Meanwhile, we also revealed that trophoblast-expressed Rbpj in EPC facilitates Mash2's transcriptional activity, promoting the specification of Tpbpa-positive trophoblasts, which differentiate into trophoblast subtypes responsible for interstitial and endovascular invasion at the later stage of placental development. Collectively, our study further shed light on the molecular network governing placental development and functions, highlighting the necessity of a spatiotemporal coordination of Rbpj signaling for normal placental morphogenesis.
\end{abstract}

\section{Introduction}

The placenta is vital to the survival and growth of the fetus before birth, since it provides the fetus with nutrients and gases during pregnancy. Any disturbance to placental development would lead to pregnancy-related

Correspondence: Shumin Wang (wangshumin@cau.edu.cn) or

Chao Wang (wangcam@cau.edu.cn) or Haibin Wang (haibin.wang@vip.163. com)

${ }^{1}$ Reproductive Medical Center, The First Affiliated Hospital of Xiamen University, 361003 Xiamen, Fujian, People's Republic of China

${ }^{2}$ Fujian Provincial Key Laboratory of Reproductive Health Research, School of Medicine, Xiamen University, 361102 Xiamen, Fujian, People's Republic of China

Full list of author information is available at the end of the article These authors contributed equally: Jinhua Lu, Weiwei Wu, Qiliang Xin Edited by G. Raschellà complications, such as preeclampsia and intrauterine growth restriction ${ }^{1-3}$. In mice, the mature placenta is composed of three distinct cellular layers: the outmost maternal decidua, derived from uterine stromal cells across which the maternal blood comes into the placenta through spiral arteries; the intermediate junction zone, through which the maternal vessels get in and out of the underlying labyrinth layer; the innermost labyrinth layer, highly branching to form large surface areas responsible for nutrient and gas exchanges between the mother and fetus $^{4}$. During placental morphogenesis in mice, the allantois derived from the extraembryonic mesoderm and the chorion developed from the extraembryonic ectoderm undergo chorioallantoic fusion at E8.5 to initiate

\section{(c) The Author(s) 2019}

(c) Open Access This article is licensed under a Creative Commons Attribution 4.0 International License, which permits use, sharing, adaptation, distribution and reproduction cc. in any medium or format, as long as you give appropriate credit to the original author(s) and the source, provide a link to the Creative Commons license, and indicate if changes were made. The images or other third party material in this article are included in the article's Creative Commons license, unless indicated otherwise in a credit line to the material. If material is not included in the article's Creative Commons license and your intended use is not permitted by statutory regulation or exceeds the permitted use, you will need to obtain permission directly from the copyright holder. To view a copy of this license, visit http://creativecommons.org/licenses/by/4.0/. 
chorioallantoic branching morphogenesis ${ }^{5,6}$, during which fetal vessels invaginate into the chorionic plate and branch with the underlying trophoblast cells to form placental labyrinth ${ }^{7}$. Simultaneously, maternal spiral arteries (SPA) delivering blood to the placenta undergo intensive remodeling, during which the endothelial cells, even regions of the smooth muscle wall, are replaced by invasive trophoblast cells. Moreover, the glycogen trophoblast cells invade interstitially into maternal decidua and form wrapping layers around spiral arteries ${ }^{8}$, contributing to spiral artery dilation ${ }^{9}$. These dynamic cellular events coordinately decrease vascular resistance of spiral arteries, thus increasing placental perfusion by maternal blood to meet the increasing needs of the fetus ${ }^{10,11}$. However, it remains largely unknown how these developmental processes are tightly coordinated to guarantee normal development and functions of the placenta.

Previous studies have demonstrated that a wide range of molecules in development-related signaling pathways is involved in regulation of placental development, including the molecules in Notch signaling ${ }^{12-14}$. Notch signaling is initiated by the release of the Notch intracellular domain (NICD) when Notch receptors are bound by its specific ligands. When translocated to the nucleus, NICD interacts directly with $\mathrm{Rbpj}$, together with Mastermind-like protein (MAML) to exert transcriptional activity ${ }^{15}$. Rbpj, the nuclear transducer of Notch signaling, is an important transcription factor highly conserved from Drosophila to human ${ }^{16}$. It has been reported that systemic deletion of Rbpj led to defective chorioallantoic fusion during early placentation ${ }^{17}$. However, it remains elusive how Rbpjmediated signaling directs normal chorioallantoic fusion. Moreover, it is still largely unknown about the precise function of Rbpj signaling during trophoblast specification, due to the early death of Rbpj-null mutants.

Employing multiple mouse models, we provided herein molecular and genetic evidence that allantoic Rbpj regulates chorioallantoic fusion and branching morphogenesis via targeting Vcam1 in a Notch-dependent manner. Meanwhile, trophoblast Rbpj facilitates Mash2's transcriptional activity to promote specification of Tpbp $\alpha$ positive trophoblasts, which differentiate into trophoblast cells responsible for interstitial and endovascular invasion at the later stage of placental development.

\section{Results}

\section{Rbpj deficiency derails normal placental development}

Our previous study has demonstrated that uterine Rbpj is essential for normal embryo development via instructing the initial embryonic-uterine orientation and ensuring normal decidual patterning in a stage-specific manner ${ }^{18}$. To further explore the pathophysiological significance of Rbpj signaling during early placental development, we first performed in situ hybridization to examine the spatiotemporal expression profile of $R b p j$ in extraembryonic tissues. At E8.0, Rbpj mRNA expression was detected in the floating allantois and chorion, and also in the ectoplacental cone (EPC), as well as trophoblast giant cells (TGC) (Fig. 1a). With the initiation and progression of chorioallantoic fusion and branching morphogenesis, Rbpj expression sustained in chorioallantoic cells at E8.5 followed by expression in placental labyrinth at E9.0-10.5, as well as in EPC and TGCs (Fig. 1a). By quantitative RT-PCR analysis, we confirmed $R b p j$ expressing in isolated allantois and chorion plus EPC (Fig. S1a). The spatiotemporal Rbpj expression indicates that Rbpj might play critical roles during chorioallantoic morphogenesis, as well as trophoblast differentiation at the fetal-maternal interface.

To determine the physiological significance of Rbpj during placental development, we employed mouse models with systemic Rbpj deletion $\left(R b p j^{-1-}\right)$ achieved by crossing

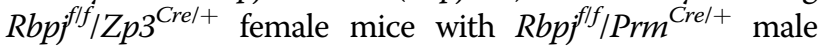
mice. At E9.5, $R b p j^{-1-}$ embryos exhibited growth-arrested and possessed pale yolk sacs with disorganized blood vessels, as well as few fetal blood cells (Fig. S1b), consistent with previous observations ${ }^{17}$. In $R_{b p f^{f / f}}$ conceptus, the allantois ( $\mathrm{Al}$, white arrowhead) attached to the chorion normally at E8.5 followed by formation of the umbilical artery and vein at E10.5. However, $R b p j^{-1-}$ mutants exhibited defective chorioallantoic attachment and fusion with the allantois (red arrowheads) failing to fuse with the chorion and appearing as a bud at E8.5 and even E9.5, leading to fetal death (Fig. 1b). HE and laminin staining further revealed that Rbpj deletion resulted in defective chorioallantoic fusion and shallow invagination of allantoic blood vessels into the chorionic ectoderm, leading to impaired chorioallantoic branching morphogenesis and labyrinth development, with no or less fetal blood vessels appearing in the placental labyrinth at E10.5 (Fig. 1c and S1c, d). These findings indicate that Rbpj deficiency derails normal chorioallantoic fusion and branching morphogenesis during placentation.

\section{$R b p j$ in allantois is indispensable for normal chorioallantoic fusion}

Chorioallantoic attachment and branching morphogenesis require intimate interaction between chorionic trophoblast and allantoic cells ${ }^{2}$. Since Rbpj was expressed in both the allantois and chorion, to ascertain the respective contribution of trophoblast versus allantoic Rbpj to chorioallantoic fusion and branching, we employed the trophoblast-specific Cyp19 $19^{\text {cre } /+}$ mouse model. In order to achieve efficient Rbpj depletion, one allele of Rbpj was first deleted systemically and then the other was deleted via Cyp $19^{\text {cre/t }}$. As shown in Fig. S2a-c, Rbpj expression was deleted specifically and efficiently in trophoblast cells, not in fetal cells $\left(\mathrm{Rbpi}^{\mathrm{fl}-} / \mathrm{Cyp} 19^{\text {cre } /+}\right.$ 


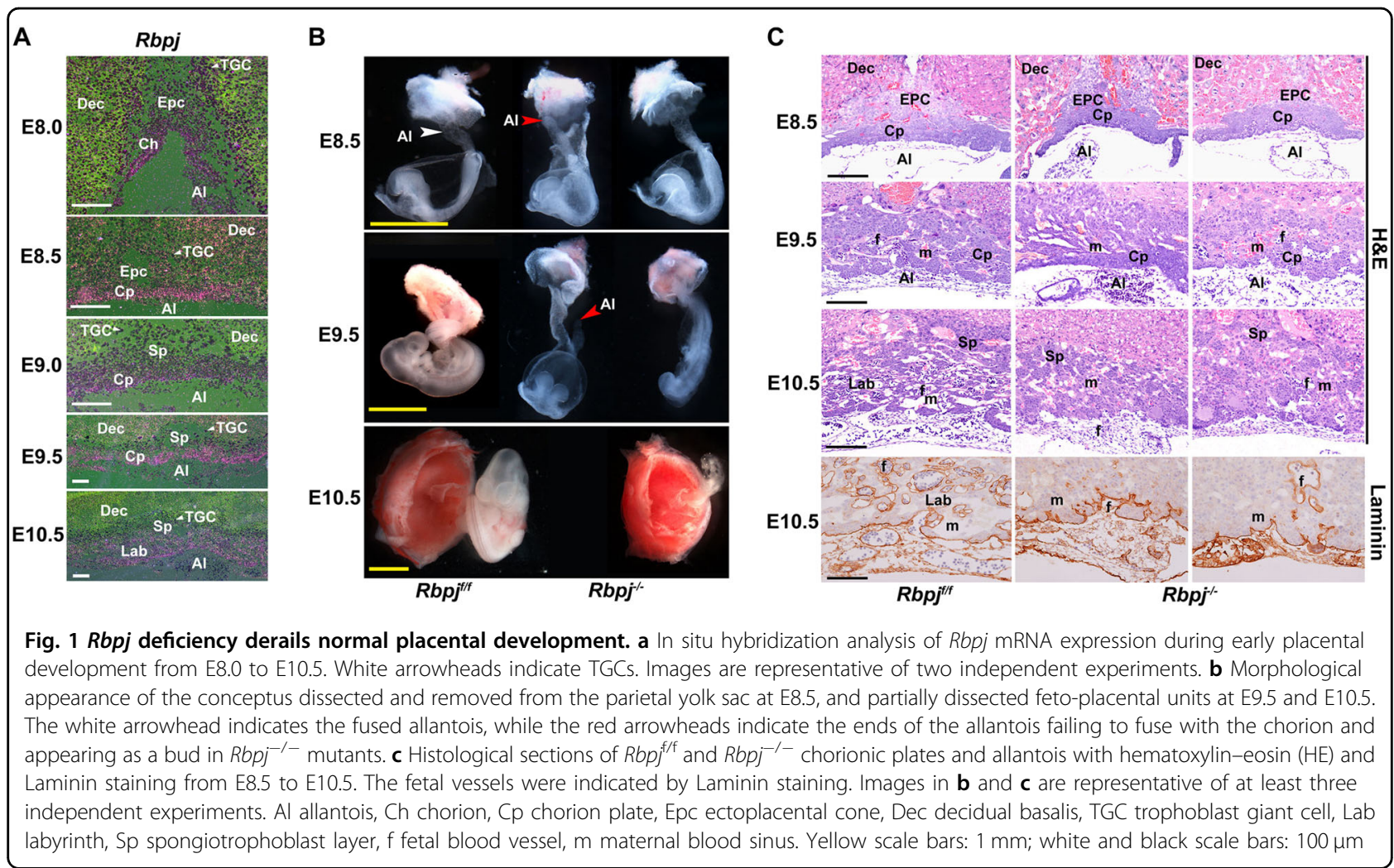

placenta). Upon trophoblast-specific Rbpj deletion, chorioallantoic attachment and fetal vascular development occurred normally in $R b p j^{f /-} / C y p 19^{\text {crel+ }}$ placentas, comparable with that of the controls $\left(R b p j^{f f f}\right.$ and $R b p j^{f /-}$ ) (Fig. $2 \mathrm{a}, \mathrm{b})$. These observations suggest that Rbpj located in the chorionic trophoblast is not required for chorioallantoic fusion and branching morphogenesis.

After chorioallantoic fusion at E8.5, chorionic trophoblast cells coordinate with the allantoic mesoderm and the underlying fetal vessels to initiate primary branching in the chorioallantoic placenta. We then analyzed the expression of marker genes involved in chorionic trophoblast differentiation in $R b p j^{f / f}$ and $R_{b p j}{ }^{-/-}$placentas with chorioallantoic fusion. At E9.0, Gcm1, which defines branching points and promotes chorionic trophoblast differentiation into syncytiotrophoblast layer II (SynTII ${ }^{19,20}$, was expressed normally at branching points in both $R b p j^{f f f}$ and $R_{b p j} j^{-1-}$ placentas. Accordingly, the expressions of Synb and Cebpo, downstream targets of Gcm1 and markers for SynT-II, were comparable in both $R b p f^{f l f}$ and $R_{b p j^{-I}}$ placentas (Fig. 2c and S2g). Moreover, the expression of Syna localized to SynT-I ${ }^{21}$, was not disturbed by Rbpj deletion (Fig. 2c and S2g). These results reinforce the notion that trophoblast-expressed $R b p j$ is dispensable for chorioallantoic trophoblast differentiation and branching morphogenesis.
Based on the aforementioned observations, we surmised that allantois-expressed Rbpj could be a vital player directing normal chorioallantoic fusion. To test this hypothesis, we first performed tetraploid aggregation assay, via which the $R_{b p j^{-1-}}$ embryos were aggregated with wildtype tetraploid embryos with EGFP expression (Fig. 3a). Tetraploid cells contribute exclusively to trophoblast cells of the placenta and endoderm of the yolk sac, rather than to allantois. As illustrated in Fig. 3b, c, while the EGFPexpressing cells derived from wild-type tetraploid embryos contributed exclusively to $R b p f^{f f f}$ placentas, as well as $\mathrm{Rbpj}^{-1-}$ placentas at E10.5, impaired chorioallantoic fusion and branching were not rescued in the chimera conceptus. These findings clearly demonstrate that allantois-expressed Rbpj is essential for normal chorioallantoic fusion and branching morphogenesis. To verify this finding, we employed Sox $2^{\text {crel+ }}$ mouse model to delete Rbpj selectively in the epiblast, and therefore in extraembryonic mesoderm-derived allantois, as well as fetal vessels in the placenta $^{22}$ (Fig. S3a, b). While growth arrest and defective chorioallantoic morphogenesis were observed in $R b p j^{f / f} /$ Sox $2^{\text {crel+ }}$ placenta (Fig. 3d and S3c), the expression of markers involved in chorionic trophoblast differentiation was unaffected (Fig. 3e). These data further reinforce the notion that allantois-expressed Rbpj is essential for normal chorioallantoic fusion and branching morphogenesis. 


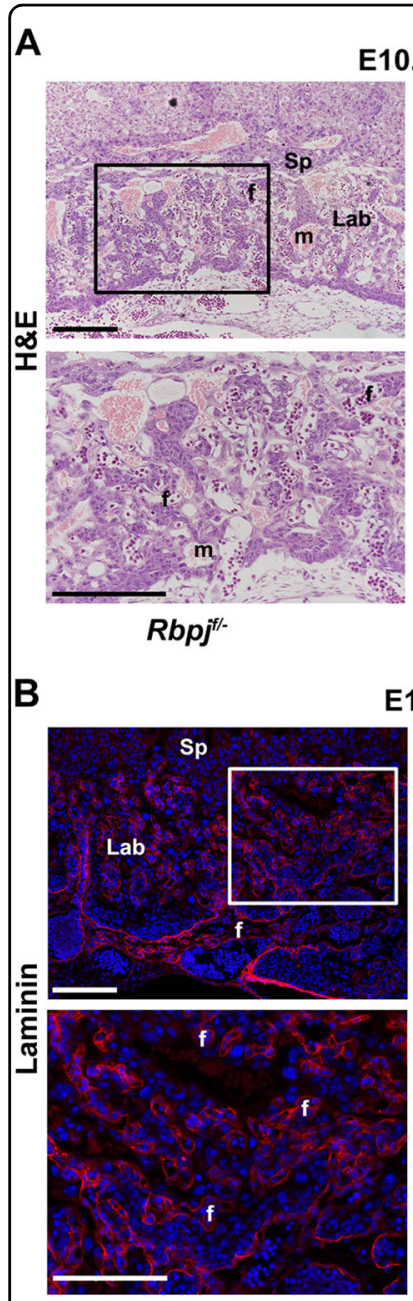

Rbpj $^{\text {f/- }}$
10.5

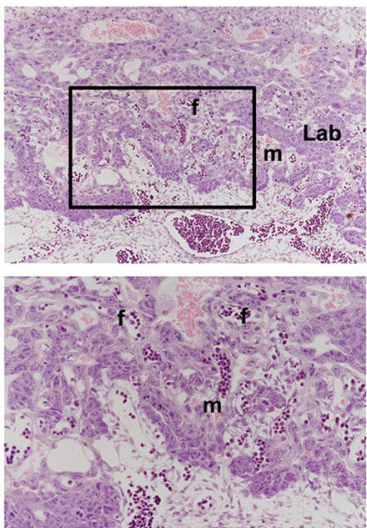

$R b p f^{f f-/ C y p 19^{c r e /+}}$

E10.5

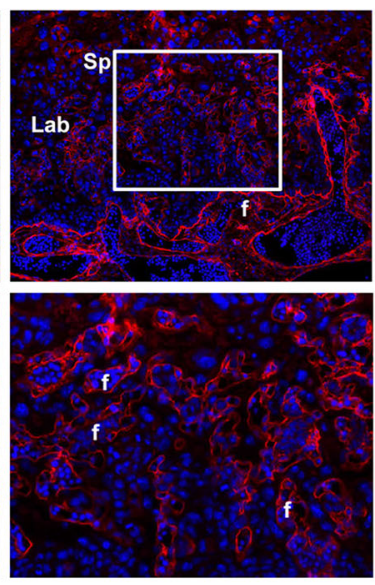

$R b p j^{f / /} / C_{y p 19}$ cre/+

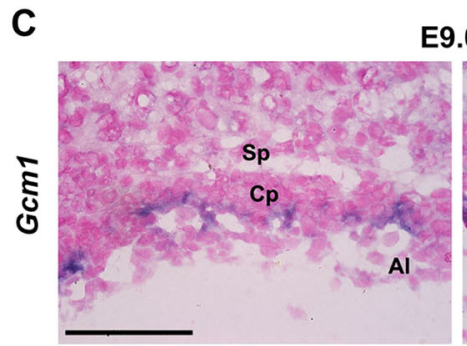

E9.0
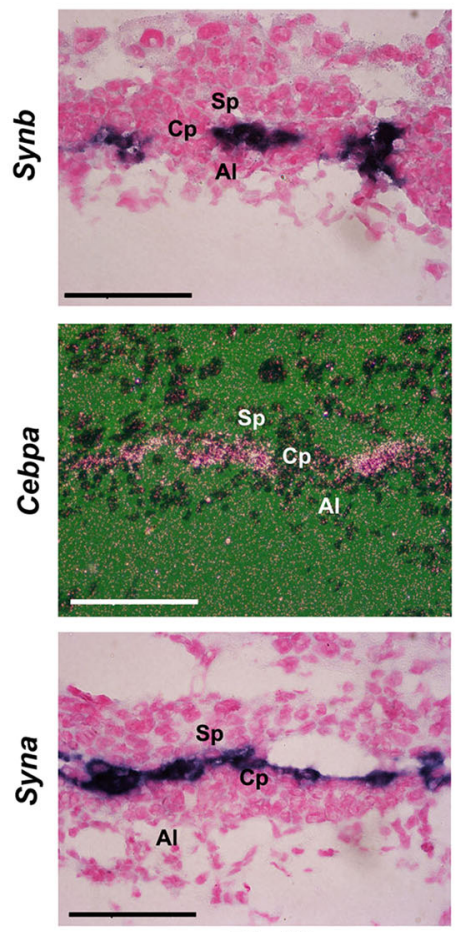

$R b p f^{f / f}$
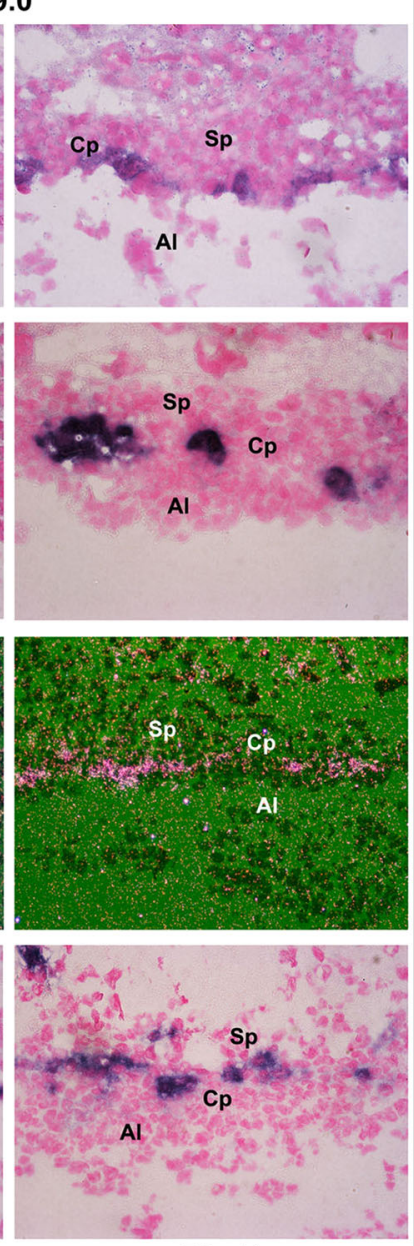

$R_{b p j^{\prime-}}$

Fig. 2 Trophoblast-expressed $\mathbf{R b p j}$ is dispensable for chorioallantoic fusion and branching morphogenesis. HE (a) and Laminin staining (b) of E10.5 placenta with Rbpj deleted specifically in placental trophoblast cells by Cyp $19^{\text {cre/+ }}$ transgenic mice model. In order to achieve efficient Rbpj deletion in trophoblast cells, we first deleted one allele of Rbpj systemically before deleting the other allele by Cyp 19 ${ }^{\text {cre/+ }}$. Cy3-labeled laminin is in red, DAPI-labeled nuclei in blue. Regions of interest are boxed in black (a) or white (b), and magnified below. $\mathbf{c}$ The differentiation of the chorionic trophoblast was revealed by the expressions of Gcml, Synb, and Cebpa (markers for SynT-II), and Syna (marker for SynT-I) in Rbpj ${ }^{\text {f/f }}$ and Rbpj ${ }^{-/-}$ chorion plate (Cp). The expression of these markers was comparable between the control $\left(R b p j^{\mathrm{f} / f}\right)$ and $R b p j$-null mutants in in situ hybridization assay. Images in a-c are representative of at least three independent experiments. Al allantois, Cp chorion plate, Lab labyrinth, Sp spongiotrophoblast layer; f fetal blood vessel; $\mathrm{m}$, maternal blood sinus. White and black scale bars: $100 \mu \mathrm{m}$

\section{Rbpj directs allantoic Vcam1 expression in a Notch- dependent manner}

Chorion-allantois fusion is based on cell-cell contact that is mediated by a variety of cell adhesions and signaling molecules. The vascular cell adhesion molecule-1 (Vcam1), expressed on the distal two-thirds of allantois, and $\alpha 4$ integrin (Itga4) localized to the chorionic basal side, lie at a pivotal step during chorioallantoic attachment and fusion, since deletion of each led to defective chorioallantoic fusion $^{23,24}$. To search for the underlying molecular basis intrinsic to allantoic defects of $R b p j$ mutants, we examined the expression of Itga4 and Vcam1 in $R b p f^{f f}$ and $R_{b p j}{ }^{-1-}$ placentas. While Itga4 expression was detectable in both the $R b p f^{f l f}$ and $R b p j^{-1-}$ basal chorions, the expression of Vcam1 almost disappeared in $\mathrm{Rbpj}^{-1-}$ placentas at E8.5 and E9.5 (Fig. 4a, b). These findings suggest a potentially functional association between Rbpj and Vcam1 during chorioallantoic attachment and branching. However, it remained unclear how allantoic Rbpj ensures normal Vcam1 expression during early placentation.

Since $R b p j$ is a transcriptional factor ${ }^{25}$, we speculated that Vcam 1 could be a direct target gene of Rbpj. In order to test this hypothesis, we searched the promoter region of Vcam 1 and found two prospective binding sites of Rbpj (Fig. 4c), located at -1006 to $-1000 \mathrm{bp}$ (VP-BS1) and 

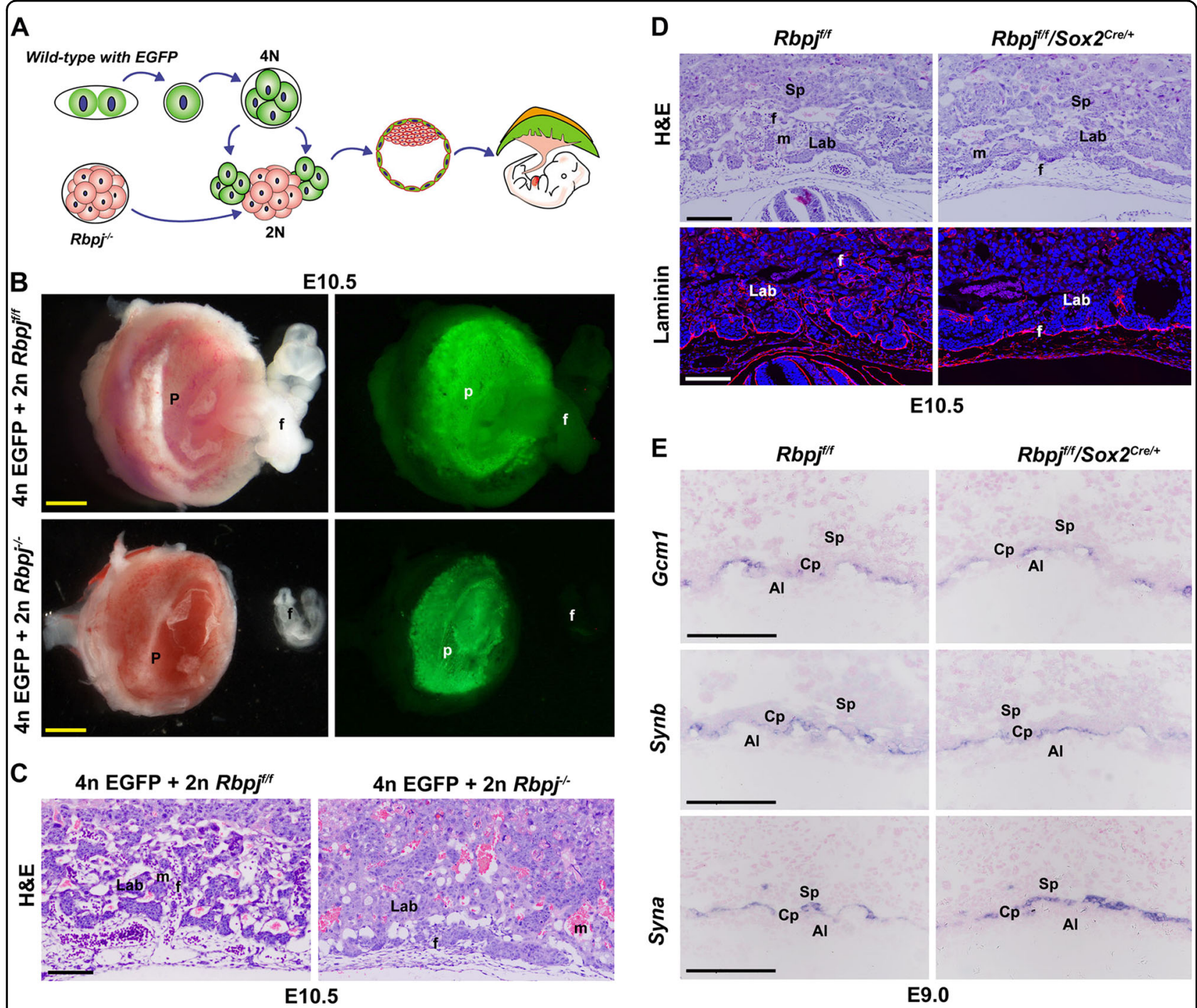

E10.5

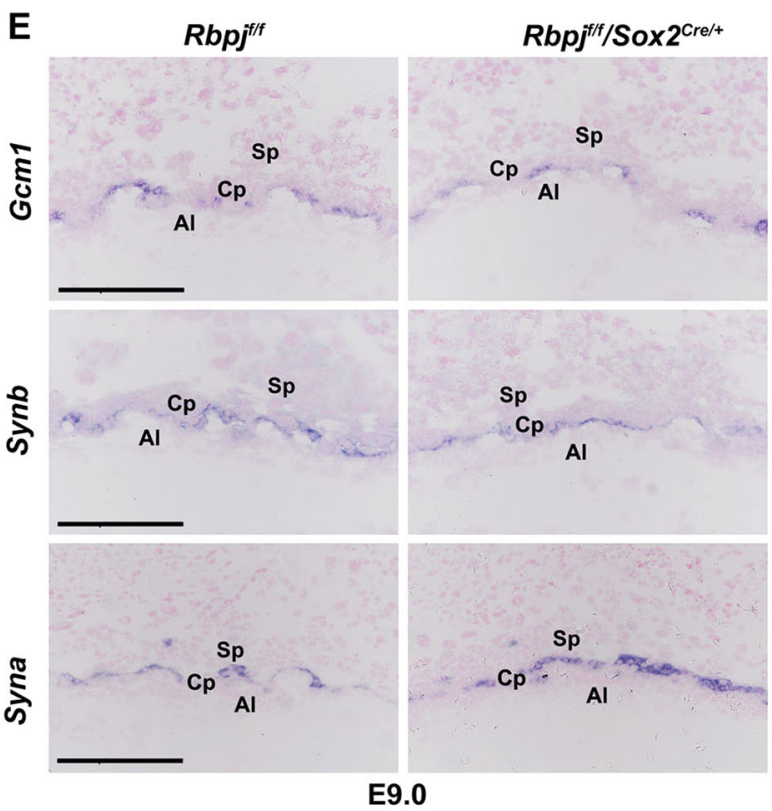

Fig. 3 Allantois-expressed $R \mathbf{b}$ pj is essential for chorioallantoic fusion and branching morphogenesis. a Diagram illustrating the procedure of tetraploid aggregation, in which one $\mathrm{Rbpj}^{-1-}$ morula aggregated with two wild-type tetraploid EGFP-expressing four-cell embryos. The tetraploid cells contribute exclusively to the trophoblast cells of the placenta, rather than the allantois. b E10.5 embryos and placentas generated by tetraploid aggregation assay. $\mathbf{c ~ H E}$ staining of E10.5 reconstructed placentas from tetraploid aggregation assay. $\mathbf{d}$ Shallow invagination of allantoic blood vessels into the chorionic ectoderm was revealed by HE and laminin staining in placentas with allantois-specific deletion of Rbpj by Sox $2^{\text {cre/+ }}$. Cy3-labeled Laminin is in red, DAPI-labeled nuclei in blue. e The differentiation of a chorionic trophoblast was revealed by the expressions of Gcm 1 and Synb (markers for SynT-II), and Syna (marker for SynT-I). By in situ hybridization, the expression of these markers was comparable between the Rbpj ${ }^{\mathrm{f} / \mathrm{f}}$ and $R b p j^{\mathrm{f} / \mathrm{f}} / \mathrm{Sox} 2^{\mathrm{cre} / \mathrm{+}}$ placentas. Images in $\mathbf{b}-\mathbf{e}$ are representative of at least three independent experiments. Al allantois, Cp chorion plate, Lab labyrinth, $\mathrm{f}$ fetal blood vessel, m maternal blood sinus, p placenta, Sp spongiotrophoblast layer. Yellow scale bars: $1 \mathrm{~mm}$; white and black scale bars: $100 \mu \mathrm{m}$

-1824 to -1818 bp (VP-BS2) upstream of the transcription start site (TSS), respectively. Chromatin immunoprecipitation (ChIP) assay using antibodies against Rbpj revealed an enriched accumulation of Rbpj at the two binding sites in E8.5 placenta (Fig. 4d). Luciferase assay further revealed that VP-BS1 was responsive to Rbpj overexpression, while its mutant form abrogating the conserved Rbpj-binding sequence (mVP-BS1) failed to respond to Rbpj signaling (Fig. 4e). Since Notch1 and
Notch2 were expressed in allantois (Fig. S4) and the phenotype of Notch1-deficient mice was similar to that of Rbpj-deficient mice ${ }^{26}$, we thus surmised that Notch1 and Notch 2 could be the prospective receptors driving Rbpj signaling to regulate Vcam1 expression during chorioallantoic fusion. Indeed, luciferase activity driven by Vcam1 promoter was increased prominently when N1ICD (Notch1) and N2ICD (Notch2) were cotransfected with Rbpj, respectively (Fig. 4e). The results suggest that 


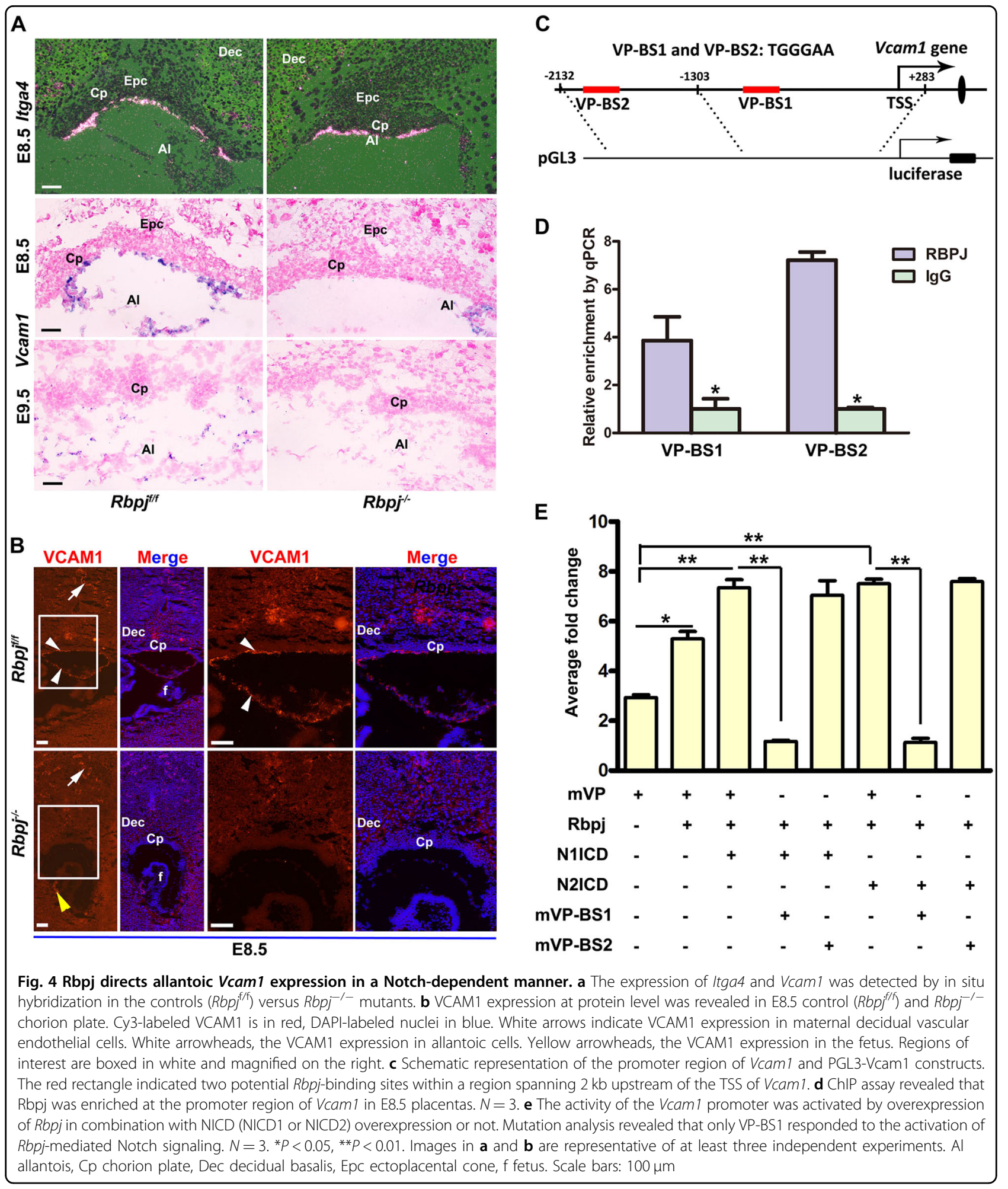

Rbpj signaling ensures normal chorioallantoic fusion and branching morphogenesis via directly inducing Vcam1 expression in a Notch-dependent manner.

To further confirm that the participation of Rbpj signaling in chorioallantoic morphogenesis is driven by canonical
Notch activation, we employed DNMAML $L^{f l+} / \mathrm{Prm}^{\text {cre/+ }}$ and $D N M A M L^{f l+} /$ Cyp $19^{\text {crel+ }}$ mouse models with haploid overexpression of dominant negative MAML (DNMAML), which blocks canonical Notch signaling ${ }^{27}$. As shown in Fig. $5 \mathrm{a}$, defective chorioallantoic development was observed in 


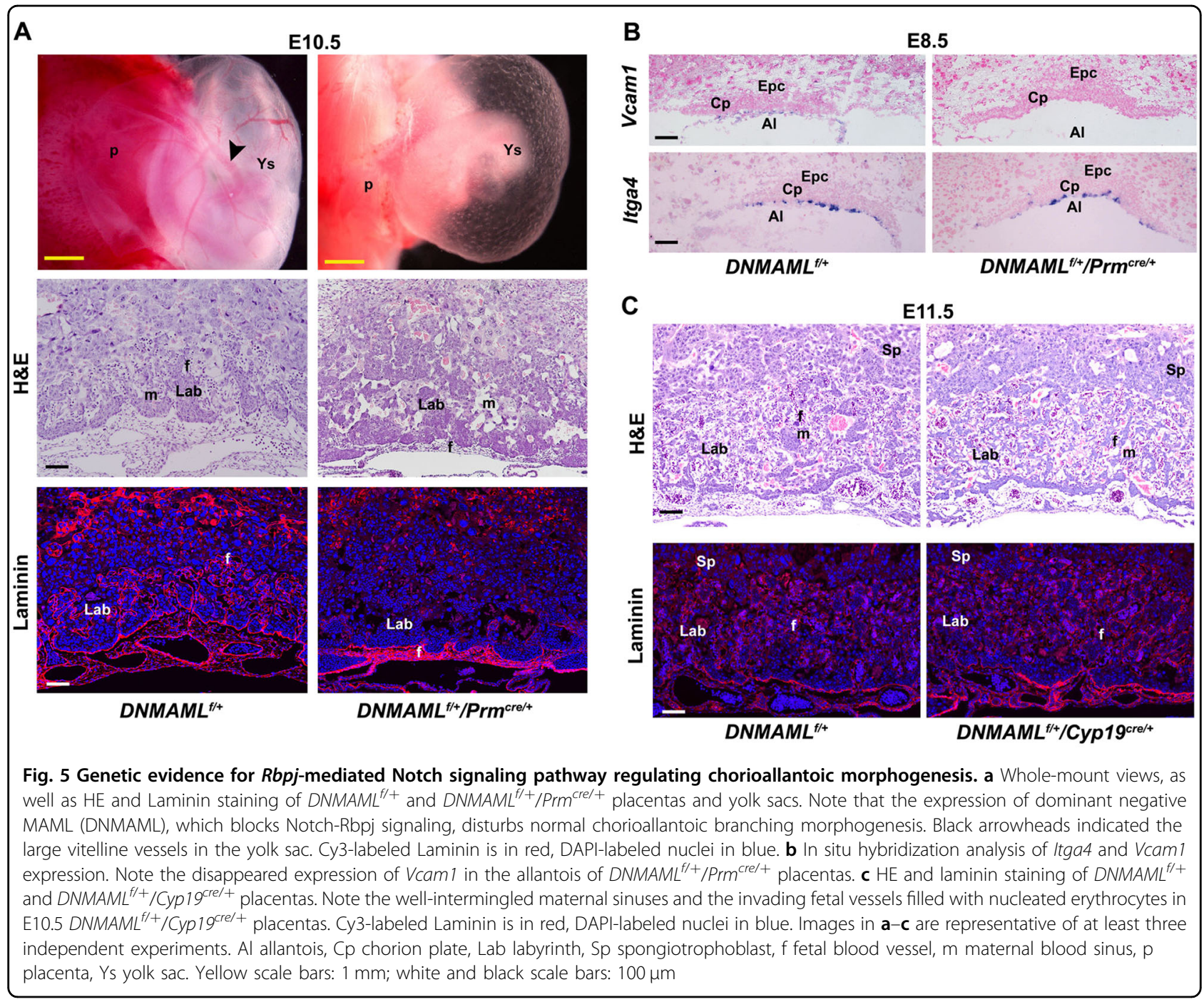

DNMAML ${ }^{f l+} / \mathrm{Prm}^{\text {crel+ }}$ placentas, similar to that of $\mathrm{Rbpj}^{-1-}$ placentas. Most importantly, Vcam1 expression was almost diminished in DNMAML $L^{f /+} / \mathrm{Prm}^{\text {cre } /+}$ allantois at E8.5, while Itga4 was expressed normally (Fig. 5b). Moreover, as expected, normal labyrinth formation was observed in DNMAML ${ }^{f l+} / C y p 19^{c r e /+}$ placenta, in which DNMAML functions specifically in trophoblast cells (Fig. 5c), consistent with the recent findings ${ }^{28}$, further demonstrating that Rbpjmediated canonical Notch signaling in chorionic trophoblast cells is dispensable for chorioallantoic attachment and branching. These data provided a new line of genetic evidence that allantoic Rbpj directs Vcam1 expression in a Notch-dependent manner.

\section{Rbpj deficiency attenuates specification of Tpbpa-positive trophoblast in EPC}

Regardless of the dispensability of Rbpj signaling in chronic trophoblast differentiation, we eventually looked into the significance of Rbpj during ectoplacental cone development, since its expression was persistent in EPC and TGCs at E8.0-10.5 (Fig. 1a). We analyzed mRNA expression of marker genes important for EPC development. The expression of heart and neural crest derivatives expressed transcript 1 (Hand1), an essential regulator of TGC specification ${ }^{29}$, was comparable between Rbpj $^{\mathrm{f} /-}$ and $R b p j^{-l-}$ placentas (Fig. 6a). This observation well correlated with the normal differentiation of Pl1-expressing TGCs in $R b p j^{-1-}$ placentas (Fig. 6a). By contrast, while the expression of achaete-scute complex homolog-like 2 (Ascl2, also Mash2), known to stimulate specification of the spongiotrophoblast lineage ${ }^{30}$, was not disturbed, the population of trophoblast-specific protein $\alpha(T p b p \alpha)$ positive cells was decreased significantly by $R b p j$ deletion (Fig. 6a). These findings suggest that Rbpj deletion attenuates specification of $T p b p \alpha$-positive trophoblast in EPC.

Since Tpbpa-positive trophoblast progenitors could differentiate into spongiotrophoblast cells, glycogen 

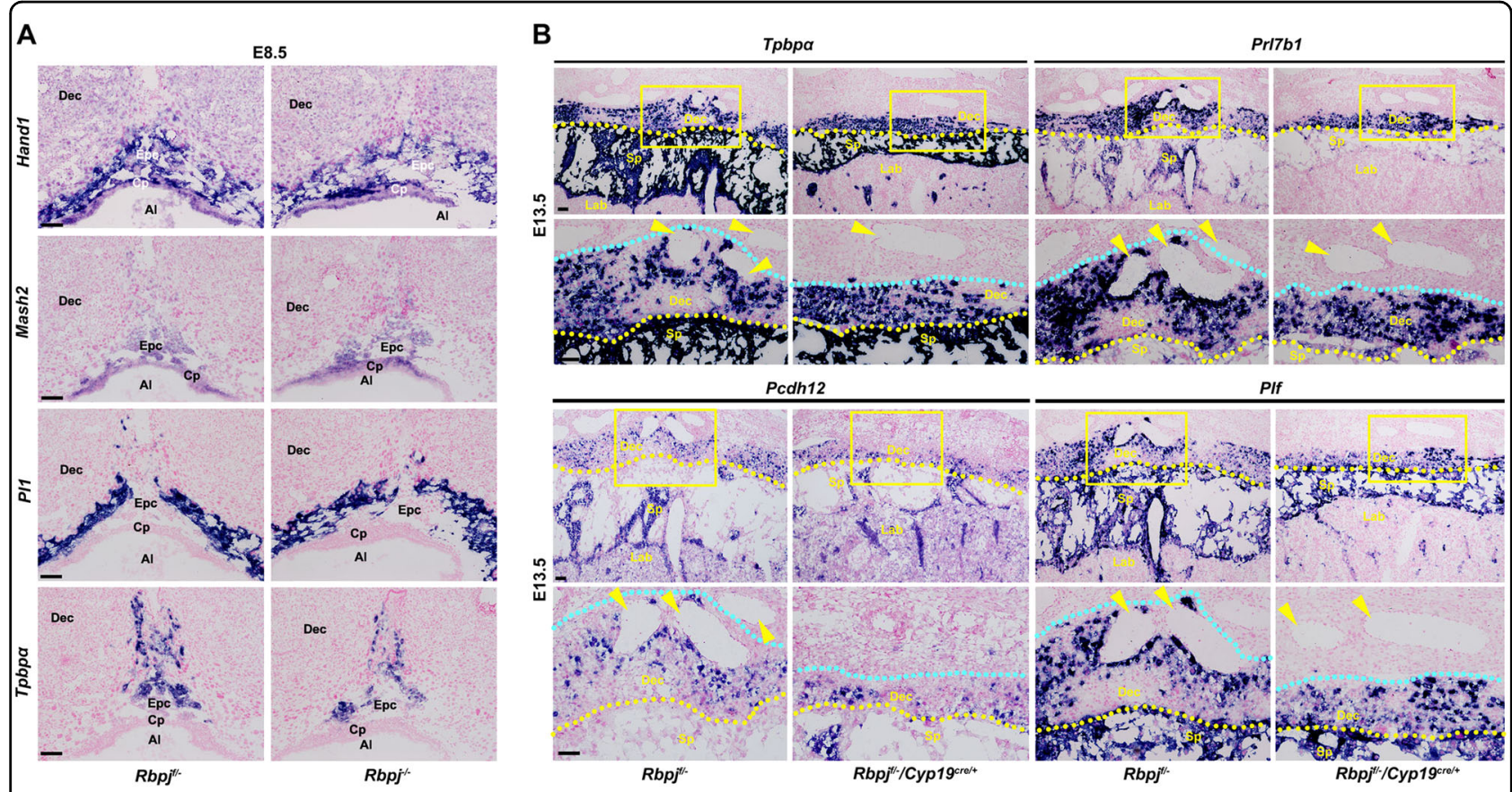

Fig. 6 Rbpj deficiency attenuates the specification of Tpbpa-positive trophoblast. a The expression of trophoblast marker genes was detected by in situ hybridization analysis in the control $\left(R b p f^{f /-}\right)$ versus Rbpj-null ectoplacental core at E8.5. The Hand1 expression was unchanged by Rbpj deletion, correlating with normal differentiation of Pl1-expressing giant cells. While the expression of Mash2 was unaffected by Rbpj deficiency, the Tpbpa-positive spongiotrophoblast cells were decreased significantly. $\mathbf{b}$ In situ hybridization analysis revealed that the invasive ability of trophoblast cells differentiated from the Tpbpa-positive trophoblast cells in the ectoplacental core was disturbed by Rbpj deletion. Prl7b1 marks both the invasive glycogen trophoblast cells and SPA-TGCs. Pcdh12 marks glycogen trophoblast cells and Plf marks SPA-TGCs. Yellow arrowheads indicate the maternal spiral arteries. Yellow dotted lines indicate the interface between the maternal decidua and the spongiotrophoblast layer, while the light blue dotted lines show the boundaries that the trophoblast invades to the maternal decidua. The distance of trophoblast invasion is between the yellow and light blue lines. Regions of interest are boxed in yellow and magnified below. Images in $\mathbf{a}$ and $\mathbf{b}$ are representative of at least three independent experiments. Al allantois, Cp chorion plate, Epc ectoplacental cone, Dec decidual basalis, Lab labyrinth, Sp spongiotrophoblast. Scale bars: $100 \mu$ m

trophoblast cells, as well as endovascular TGCs that invade into the maternal decidua and SPA, respectively, beyond mid-gestation ${ }^{31,32}$, we wonder whether attenuated specification of $T p b p \alpha$-positive trophoblast would hamper the functions of mature placenta, particularly trophoblast invasion into the maternal decidua and SPA. Indeed, the E13.5 fetuses with $R b p f^{f /-} /$ Cyp $19^{\text {crel+ }}$ placentas were a little pale, compared with those of control placentas (Fig. S5a). Moreover, the weight of the fetuses with $R b p f^{f f-} / C y p 19^{\text {crel+ }}$ placenta was decreased significantly, while the placental weight was unaffected (Fig. S5b). We next analyzed the differentiation of Tpbp $\alpha$-positive trophoblast in E13.5 placentas. As illustrated in Fig. 6b, Tpbpo-positive spongiotrophoblast population was reduced significantly upon Rbpj deficiency $\left(R b p f^{f /-} / C y p 19^{c r e /+}\right)$. More importantly, the population and invasion of glycogen trophoblasts expressing Prl7b1 and Pcdh12 were reduced substantially in Rbpi $^{\text {fl- }} /$ Cyp $19^{\text {crel+ }}$ placentas (Fig. 6b). Moreover, the differentiation and invasion of Prl7b1-positive and Plf-positive TGCs were disturbed by Rbpj deletion (Fig. 6b). These results indicate that intact $\mathrm{Rbpj}$ signaling is essential for normal specification of Tpbp $\alpha$-positive trophoblast cells.

\section{Rbpj facilitates Mash2 to promote specification of Tpbpa- positive trophoblast}

Previous evidence disclosed that Notch signaling regulated Mash2 through hairy/Enhancer of split (Hes) genes and Groucho genes (Tle) in Drosophila ${ }^{33,34}$, implying a potential coupling of Notch signaling with Mash2 during the specification of Tpbp $\alpha$-positive cells. To reveal the underlying molecular mechanism governing normal specification of $T p b p \alpha$-positive trophoblast driven by Rbpj signaling, we further employed cultured trophoblast stem (TS) cell lines (Fig. S6a). Rbpj deletion did not perturb the stemness of trophoblast cells, since $R b p j^{-1-}$ trophoblast stem cells have been established successfully. We found that while the expression of Mash2 was not disturbed, Tpbpa expression was decreased significantly, but not disappeared in Rbpj-deficient TS cells undergoing default differentiation in culture (Fig. 7a), consistent with in vivo observations (Fig. 6a). In addition, Mash2 knockdown by 


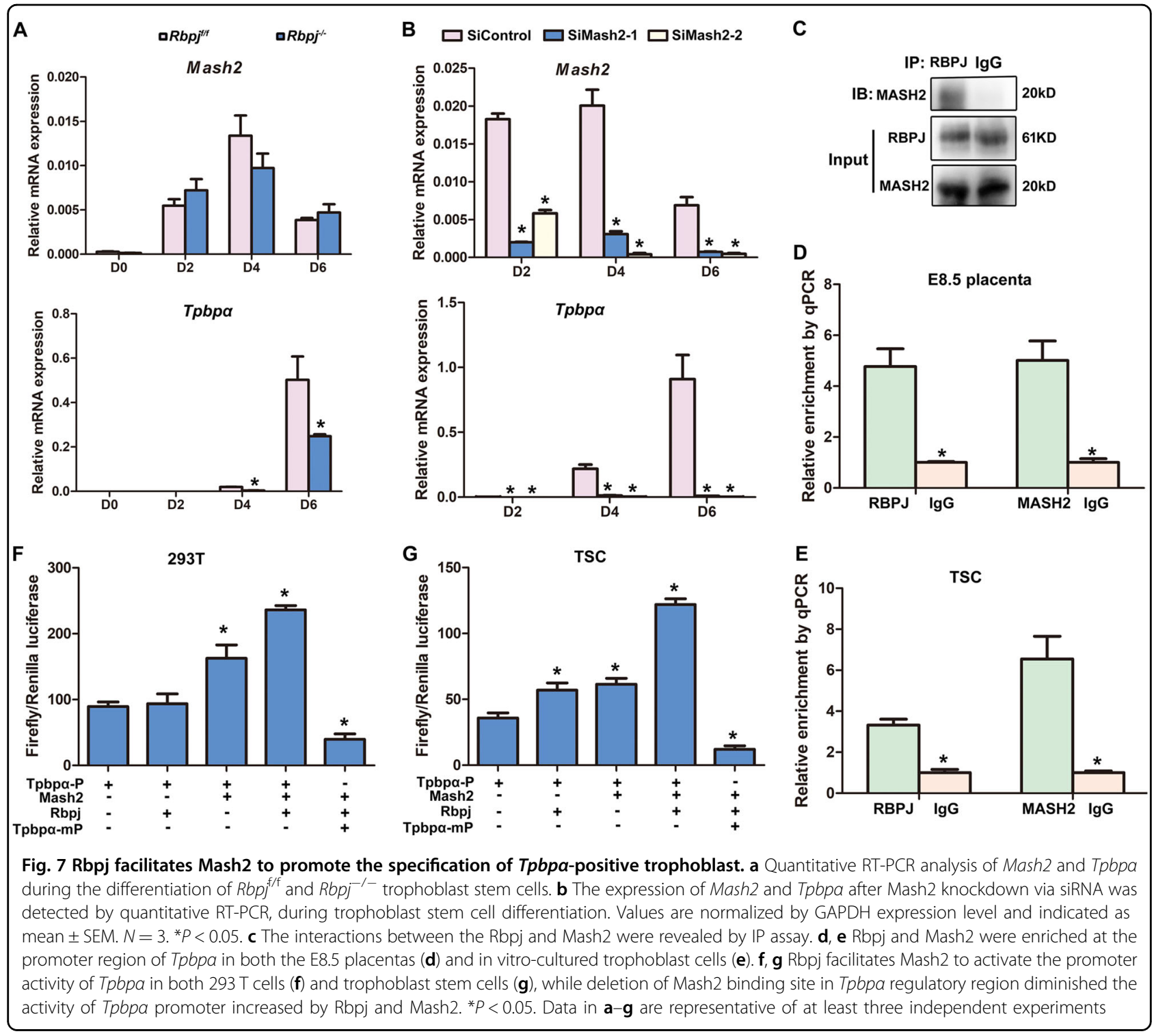

siRNA almost diminished Tpbpa expression (Fig. 7b), similar to the findings in Mash2 knockout mice ${ }^{30}$. These findings suggest that Rbpj might facilitate the functions of Mash2 during specification of Tpbpo-positive trophoblast. Co-immunoprecipitation analysis further revealed physical interaction between Mash2 and Rbpj in trophoblast cells (Fig. 7c). Since Rbpj and Mash2 are both transcription factors, we performed ChIP assay using antibodies against Mash2 and Rbpj, respectively. We found an enriched accumulation of both Mash2 and Rbpj in a trophoblast-specific regulatory region of $T p b p \alpha$, a 340-bp region that is $3.7 \mathrm{~kb}$ upstream of the TSS of $T p b p \alpha^{35}$, in both E8.5 placenta (Fig. 7d) and TSC (Fig. 7e). These data demonstrate a functional association of Rbpj and Mash2 during the specification of $T p b p \alpha$-positive trophoblast cells.
Regarding the physiological significance of Mash2-Rbpj interaction, we employed luciferase assay to assess the activity of Tpbpa promotor in both HEK $293 \mathrm{~T}$ cells and TS cells. While Mash2 increased luciferase activity significantly, Rbpj alone exhibited no influence on luciferase activity in HEK 293 T cells (Fig. 7f), which have extremely low expression of endogenous Mash2. Importantly, cotransfection of Mash2 and Rbpj increased the activity of $T p b p \alpha$ promoter significantly (Fig. $7 \mathrm{f}$ ). In consistence, a similar phenomenon was observed in cultured TS cells undergoing differentiation; besides that, Rbpj alone was able to promote the activity of Tpbpa promoter (Fig. 7g), since TS cells expressed endogenous Mash2. Moreover, employing in silico analysis of the trophoblast-specific regulatory region of $T p b p \alpha$, we found the binding site of Mash2, but not that of Rbpj. When the binding site of 

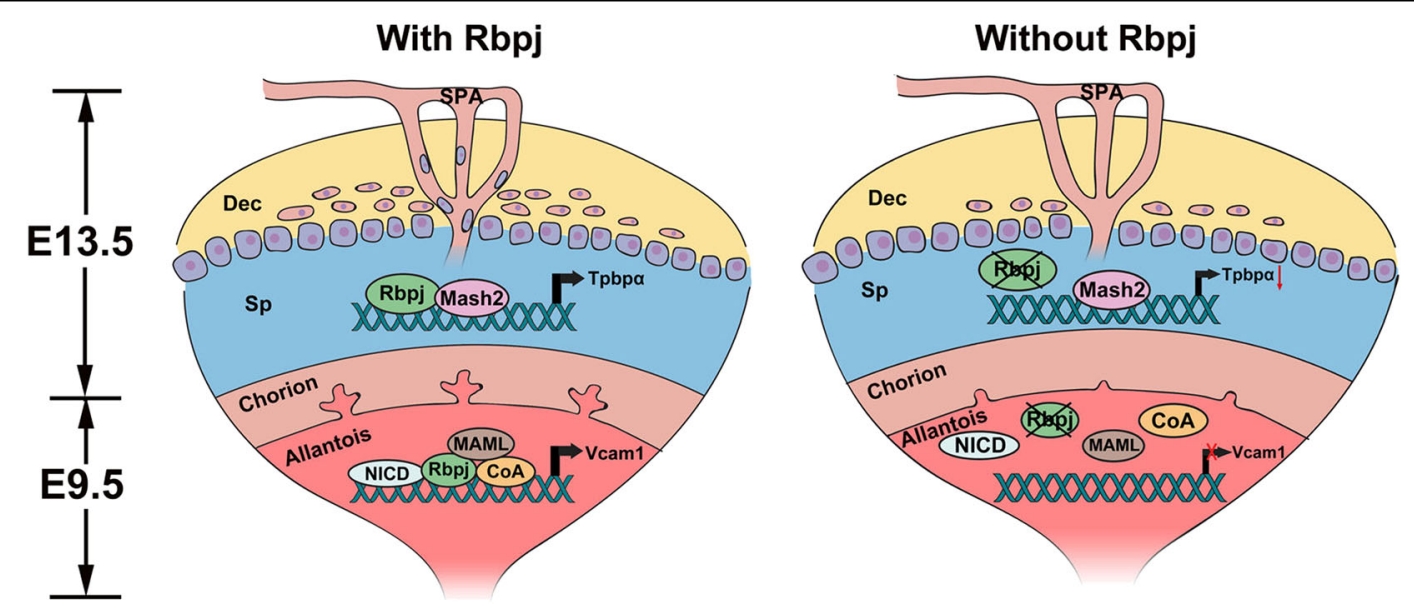

Fig. 8 Diagram illustrating the functions of RBPJ-mediated signaling during chorioallantoic development and trophoblast differentiation in EPC (Sp). In the presence of Rbpj (with Rbpj), Rbpj regulates the expression of $V_{c a m} 1$ in the allantois through a Notch-dependent manner to ensure normal chorioallantoic branching morphogenesis, and facilitated Mash2 in EPC (Sp) to promote the specification of Tpbpa-positive

trophoblast cells simultaneously. However, failed chorioallantoic branching and defective trophoblast differentiation in EPC (Sp) were observed in the absence of Rbpj (without Rbpj). Dec decidual basalis, Sp spongiotrophoblast layer, SPA spiral arteries

Mash2 was deleted, the activity of Tpbpa promoter increased by Rbpj and Mash2 was decreased significantly (Fig. 7f, g). In addition, while endogenous Tpbpa expression was promoted by Rbpj alone in the presence of endogenous Mash2, its expression was further increased synergistically by Mash2 and Rbpj overexpression in differentiated trophoblast cells (Fig. S6b, c). These data demonstrate that Rbpj facilitates Mash2 to promote the specification of $T p b p \alpha$-positive trophoblast.

\section{Discussion}

Placenta acts as the interface between the mother and fetus for the exchanges of nutrients, gases, and wastes. During placental development, fetal vessels grow into the labyrinth via chorioallantoic branching morphogenesis. Simultaneously, the maternal blood enters the placenta through SPA remodeled by TGCs and facilitated by invasive glycogen trophoblast cells, and forms blood sinus in the placental labyrinth layer. We provided herein genetic and molecular evidence that allantoic Rbpj guides normal chorioallantoic fusion and branching morphogenesis by targeting Vcam1, while trophoblast Rbpj facilitates the functions of Mash2 during specification of $T p b p \alpha$-positive trophoblast, which are important for the differentiation and invasion of SPA-TGCs and glycogen trophoblast cells during placental development (Fig. 8). These findings highlight the necessity of a spatiotemporal coordination of Rbpj signaling for normal placental morphogenesis.

Chorioallantoic fusion is the first step for fetal vessels to invaginate into the chorion and followed by further branching of fetal vessels in the placental labyrinth. In this study, Rbpj-mediated signaling regulates chorioallantoic fusion via Vcam1 in a Notch-dependent manner, highlighting the necessity of Rbpj-Vcam1 signaling during chorioallantoic fusion ${ }^{23,36}$. Moreover, we further demonstrated that this signaling pathway might also function during chorioallantoic branching beyond the initial fusion, since branching failed to develop further into the labyrinth in the absence of Rbpj or Vcam1. We found that only Vcam1 expression in allantois and allantois-derived fetal vessels was disturbed, but not that in fetuses with Rbpj deletion (Fig. 4b), suggesting that regulation of Vcam1 expression is tissue specific. Moreover, Rbpjdeficient mice with defective chorioallantoic fusion also suffered from impaired labyrinth development, with fetal vessels failing to extend and invaginate further into the labyrinth layer. We also found that cultured allantois exhibited decreased vascular remodeling, when Rbpj was deleted or Notch signaling was inhibited by DAPT (Fig. S7). These findings reinforce the content that allantoic Notch-Rbpj-Vcam1 signaling is also essential for remodeling of placental fetal vessels beyond the initial fusion $^{7,24,37}$. In addition, Dll4 was reported to be expressed in fetal vascular endothelial cells of allantois and subsequent umbilical arteries. Haploid insufficiency of Dll4 exhibits similar phenotypes to that of Rbpj-null mice $^{38-40}$, suggesting that Dll4 is a potential upstream driver of Rpbj-Vcam1 signaling governing normal vascular remodeling and the maintenance of fetal vessels during chorioallantoic morphogenesis.

During pregnancy, maternal blood flow through the placenta increases dramatically, largely due to significant vasodilation of blood vessels ${ }^{10}$. In mice, TGCs invading utero-placental SPA and glycogen trophoblast cells invading interstitially into the decidua, are associated with 
the remodeling of maternal SPA into dilated, inelastic tubes to increase maternal blood flow to the placenta ${ }^{32}$. It has been shown that TGCs and glycogen trophoblast cells are differentiated from progenitor cells localized within the ectoplacental cone, as well as the spongiotrophoblast layer later, and expressing spongiotrophoblast-specific gene $T p b p \alpha^{31}$. In our work, we found that decreased Tpbpa expression by Rbpj deletion led to defective invasion of SPA-TGCs and glycogen trophoblast cells. This may help explain why fetal weight was not perturbed at E10.5, but was decreased at E13.5, in the presence of trophoblast-specific Rbpj deletion. Fetal weight at E10.5 was not disturbed, maybe due to normal development of placental labyrinth after Rbpj deletion. However, with rapid fetal growth since E10.5, the fetuses failed to get enough nutrients to meet their increasing needs, might attribute to impaired remodeling of maternal SPA and insufficient placental perfusion upon Rbpj deletion, leading to decreased fetal weight at E13.5.

Although it has been reported that Mash2 is a key determinant for specification of Tpbpo-positive trophoblast cells ${ }^{41}$, the mechanism controlling specialization of Tpbpo-positive cells remains largely unknown. In our study, we demonstrated that Rbpj facilitates Mash2 to promote $T p b p \alpha$ expression and specialization of Tpbp $\alpha$ positive cells. These findings provide a new line of evidence regarding the regulation of $T p b p \alpha$-positive trophoblast cells specification. Moreover, the findings that decreased Tpbpa expression upon Rbpj deletion led to defective invasion of SPA-TGCs and glycogen trophoblast cells are similar to the phenotypes of mice with conditional deletion of Notch2 via Tpbpo-Cre ${ }^{42}$. Although Notch2 is involved in the process during which Tpbpopositive cells differentiate into invasive SPA-TGCs and glycogen trophoblast cells ${ }^{42}$, it seems that Notch2 does not participate in Rbpj-mediated signaling responsible for specification of $T p b p \alpha$-positive trophoblast cells in EPC, since the population of Tpbp $\alpha$-positive cells was not disturbed in Notch2-deficient placentas ${ }^{43}$. Moreover, it has been shown that Rbpj could also function in a Notchindependent manner ${ }^{18,44-46}$, and trophoblast-specific inhibition of canonical Notch signaling did not disturb placental development ${ }^{28}$. We also found that Rbpj had no binding sites in the trophoblast-specific regulatory region of Tpbpa. Therefore, Rbpj-mediated signaling might regulate specification of $T p b p \alpha$-positive trophoblast cells in a noncanonical manner. In addition, it has been reported that trophoblast cells expressing Prdm/Blimp1 gave rise to SPA/endovascular TGCs, canal TGCs, and glycogen trophoblast cells, and Tpbpa expression was unaffected in the absence of Blimp $1^{47}$, suggesting that Blimp1 might function after the specification of Tpbp $\alpha$ positive trophoblast cells, and Rbpj signaling may also regulate specification of Blimp1-positive cells.
In summary, our study further shed light on the molecular network governing placental development and functions, and the findings might have clinical relevance, since abnormal Notch signaling contributes to pathogenesis of gestational diseases with aberrant placentation and trophoblast function in women ${ }^{14,42}$.

\section{Materials and methods \\ Animals \\ $R b p j^{\text {loxplloxp }}\left(R_{\left.b p j^{\mathrm{f} / \mathrm{f}}\right)}\right.$ mice, DNMAML $L^{f / f}$ mice, and} Cyp $19^{\text {cre/+ }}$ transgenic mice were generated as previously described ${ }^{27,48,49}$. Enhanced green fluorescent protein $(E g f p)^{T g /+}$, Rosa26-LacZ ${ }^{\mathrm{f} / \mathrm{f}}$, Prm $^{\text {cre } /+}$, ZP3-cre ${ }^{\text {cre } /+}$, and So $x 2^{\text {cre/+ }}$ transgenic mice were obtained from Jackson Laboratory. $\mathrm{Rbpj}^{\mathrm{f} / \mathrm{f}}$ mice were mated with $\mathrm{Zp} 3^{\mathrm{Crel+}}$ and $\mathrm{Prm}^{\text {cre/+ }}$ to achieve systemic Rbpj deletion. $R b p j^{\mathrm{f} / \mathrm{f}}$ mice were mated with Sox $2^{\text {crel+ }}$ and Cyp $19^{\text {crel+ }}$ transgenic mice to get conditional deletion of Rbpj in the epiblast and trophoblast derivates, respectively. $D N M A M L^{f l f}$ mice were mated with $\mathrm{Prm}^{\mathrm{Crel+}}$ transgenic mice and Cyp19 $\mathrm{Crel}$ + transgenic mice to silence canonical Notch signaling in the global and trophoblast-derived cells, respectively. Females were mated with fertile males of the same strain to induce pregnancy, and mice with virginal plus were considered as embryonic day 0.5 (E0.5). Mice were housed in the animal care facility of the Xiamen University according to the guidelines for the care and use of laboratory animals.

\section{Histological analysis and immunostaining}

For hematoxylin and eosin staining, isolated implantation sites or whole dissected placental tissues were collected at various stages of gestation and fixed in 10\% neutral buffer formalin. Then tissues were dehydrated and embedded in paraffin wax before being cut into $5-\mu \mathrm{m}$ sections. For immunohistochemistry analysis, the rehydrated sections were incubated with RBPJ (1:200, Cell Signaling Technology) and Laminin (1:200, Sigma) antibodies, respectively. A Histostain-plus Kit (Zhongshan Golden Bridge Biotechnology) was used to visualize the antigen. For immunofluorescence, antibodies specific to Laminin (1:500, Sigma) and Vcam1 (1:500, Abcam) and secondary antibodies conjugated with $\mathrm{Cy} 3$ dyes (Jackson ImmunoResearch Laboratories) were used to stain rehydrated sections. Immunofluorescence images were captured in a Zeiss LSM 780 confocal scanning laser microscope.

\section{In situ hybridization}

In situ hybridization was performed on cryosections as previously described ${ }^{21,50}$. Briefly, frozen sections $(10 \mu \mathrm{m})$ were mounted onto poly-L-lysine-coated slides and fixed in $4 \%$ paraformaldehyde solution in PBS at $4{ }^{\circ} \mathrm{C}$. After prehybridization, sections were hybridized at $45^{\circ} \mathrm{C}$ for $4 \mathrm{~h}$ 
in $50 \%$ formamide buffer containing ${ }^{35} \mathrm{~S}$ or digoxygenin (DIG)-labeled sense or antisense RNA probes. The primers for probe production are listed in Table S1. After hybridization, sections were incubated with RNase A $(10 \mathrm{mg} / \mathrm{ml})$ at $37^{\circ} \mathrm{C}$ for $20 \mathrm{~min}$, and RNase A-resistant hybrids were detected by autoradiography, using liquid emulsion or NBT/BCIP chromogenic agent. Sections hybridized with the sense probes served as negative controls.

\section{Tetraploid aggregation assay}

Tetraploid aggregation chimeras were generated as described previously with some modifications ${ }^{51}$. In brief, wild-type tetraploid embryos were generated by electrofusion of two-cell embryos derived from EGFP intercrosses. Fused embryos were cultured overnight in KSOM medium and developed to form four-cell embryos before the removal of zona pellucida with acidic Tyrode solution. Diploid Rbpj-null embryos at eight-cell or morula stage were collected at E2.5. Each diploid embryo with zona pellucida removed was aggregated with two tetraploid embryos. Aggregated chimeric embryos were allowed to develop into the blastocyst stage and then transferred into the pseudopregnant uteri of wild-type females. Chimeric embryos were dissected at E10.5. Both the placenta and embryo proper were visualized for EGFP under a dissecting microscope and subsequently fixed for staining with hematoxylin-eosin. The placental and embryo tissue was used as a DNA source for genotyping of diploid embryos.

\section{Quantitative real-time PCR}

Total RNA was extracted from embryonic tissues or trophoblast cells using TRIzol reagent (Invitrogen), and $1-3 \mu \mathrm{g}$ of total RNA after DNase treatment was used to synthesize cDNA. Quantitative RT-PCR analysis of different genes was performed using ABI QuantStudio 5 Real-Time PCR system, according to the manufacturer's instructions. Assays were performed at least three times with each in duplicate. All the primers for real-time PCR are listed in Table S1.

\section{Plasmid construction, transfection, and luciferase assay}

The promoter region of Vcam 1 gene $(2 \mathrm{~kb}$ upstream relative to the transcription start site) with VP-BS1 and VP-BS2 wild-type versus mutant vectors (TGGGAA to TCTCAA) was generated using mouse genomic DNA as template and cloned into pGL3-basic vectors (Promega). Similarly, pGL3-basic vectors containing a trophoblastspecific regulatory region of $T p b p \alpha$, a 340-bp region that has been reported and is $3.7 \mathrm{~kb}$ upstream of the TSS of Tpbpa ${ }^{35}$, were constructed from genomic DNA similarly. Mutations of the Mash2 binding site were achieved by Fast Mutagenesis System (Stratagene). The coding sequence region for the intracellular domain of Notch1 and Notch2 were amplified from the mouse placenta and cloned into the $\mathrm{pCDH}$ expression vector. In addition, the coding sequence region of $\mathrm{Rbpj}$ and Mash2 was constructed with pEGFP-N1 and pcmv-myc expression vectors, respectively. Primers used are listed in Table S1.

HEK293T cells were maintained in Dulbecco's Modified Eagle Medium (DMEM) (Hyclone) containing 10\% fetal calf serum, $1 \mathrm{mM}$ sodium pyruvate, $2 \mathrm{mM}$ glutamine, $50 \mathrm{IU} / \mathrm{ml}$ penicillin, and $50 \mu \mathrm{g} / \mathrm{ml}$ streptomycin. All constructs were transiently transfected into HEK293T cells in 96-well plates, using Lipofectamine 2000 reagent (Invitrogen), according to the manufacturer's instructions. pRLTK, the internal control plasmid expressing Renilla luciferase (Promega), was cotransfected into the cells to normalize firefly luciferase activity of the reporter plasmids. Transfected cells were harvested after $24 \mathrm{~h}$. Luciferase assay was performed by Dual-Luciferase Reporter System (Promega) according to the manufacturer's instructions. Assays were performed at least three times with each in duplicate.

\section{Allantois culture and immunostaining}

Briefly, $R b p j^{\mathrm{f} / \mathrm{f}}$ and $R b p j^{-/-}$pregnant females were killed by cervical dislocation at E8.0. Allantois could be clearly identified at the end of PS in the exocoelom after wiping off the Reichert's membrane. The floating allantoises were excised at the basal part, rinsed in cold CZB and culture medium three times, respectively, transferred to the 24well Petri dish that has been coated with fibronectin, and then cultured for $24 \mathrm{~h}$. The allantois explants were fixed in $4 \%$ PFA, permeabilized in $0.2 \%$ Triton $\mathrm{X}-100$, and incubated with rat anti-PECAM1 (1:500, BD) overnight at $4{ }^{\circ} \mathrm{C}$. Then they were washed in $0.1 \%$ BSA-PBS, incubated with the Cy3-labeled secondary antibody.

\section{Co-immunoprecipitation (Co-IP) and chromatin immunoprecipitation (ChIP)}

Protein samples $(1 \mathrm{mg})$ from E8.5 placentas or trophoblast cells lysated by mild buffer $(25 \mathrm{mM}$ Tris, $150 \mathrm{mM}$ $\mathrm{NaCl}, 1 \mathrm{mM}$ EDTA, 1\% Nonidet P-40, and 5\% glycerol; $\mathrm{pH}$ 7.4) were used for Co-IP experiments. Antibodies to Rbpj (Santa Cruz Biotechnology), Mash2 (Merck) were used. Protein A agarose beads (Thermo-Fisher Scientific) were washed and incubated with protein lysates overnight at $4{ }^{\circ} \mathrm{C}$. Immunoprecipitated proteins were separated by SDS/PAGE and detected by immunoblotting using the respective antibodies. ChIP analysis was performed according to the instructions of the ChIP Assay Kit (Millipore). In brief, E8.5 placentas or trophoblast cells were collected and suspended in $1 \%$ formaldehyde (Sigma)-PBS solution for cross-linking. After the crosslinking was terminated, cell pellets were lysed and sheared by sonication, until the average length of DNA was 
$\sim 500$ bp as evaluated by agarose gel electrophoresis. The sheared chromatin fragments were incubated with the RBPJ (Santa Cruz Biotechnology) or Mash2 (Millipore) antibodies overnight at $4{ }^{\circ} \mathrm{C}$. Normal goat IgG (Santa Cruz Biotechnology) was used as a negative control for nonspecific immunoprecipitation. After washing, the beads were suspended in elution buffer and the precipitated protein/DNA complexes were eluted from the antibodies/ beads. Then the chromatin was subjected to cross-link reversal and DNA was purified by phenol/chloroform extraction and ethanol precipitation. Specific primers listed in Table S1 were used to detect immunoprecipitated chromatin fragments and input chromatin.

\section{Statistical analysis}

Statistical analysis was performed with SPSS11.5 program. Comparison of means was performed using the independent-samples $t$ test. Each experiment was performed at least three times with each in duplicate. Data were shown as means \pm SEM. ${ }^{*} p<0.05$; ${ }^{* *} 0.01<p<0.05$.

\section{Acknowledgements}

We are grateful to Drs. Tasuku Honjo, Warren S. Pear, and Gustavo Leone for their generosity in providing us the Rbpj f/ff, DNMAML ${ }^{\text {f/f }}$ mice, and Cyp $19^{\text {cre/ }++}$ mouse models, respectively. We also like to thank Jingtao Qiu for assistance in in situ hybridization. This work was supported in parts by National Key R\&D Program of China (2017YFC1001402 to H.W., 2018 YFC1004102 to J.L.), National Natural Science Foundation of China (81490744 to H.W., 31600945 to J.L., and 31701016 to J.W.), Fujian Natural Science Foundation (2017J01071 to J.L.), the Fundamental Research Funds for the Central Universities (20720180041 to J.L.), and Foundation from Key Laboratory of Reproduction Regulation of NPFPC (2017KF01 to J.L.). The funders had no role in study design, data collection and analysis, decision to publish, or preparation of the paper.

\section{Author details}

${ }^{1}$ Reproductive Medical Center, The First Affiliated Hospital of Xiamen University, 361003 Xiamen, Fujian, People's Republic of China. ${ }^{2}$ Fujian Provincial Key Laboratory of Reproductive Health Research, School of Medicine, Xiamen University, 361102 Xiamen, Fujian, People's Republic of China. ${ }^{3}$ Anti-aging \& Regenerative Medicine Research Institution, College of Life Sciences, Shandong University of Technology, 255049 Zibo, Shandong, People's Republic of China. ${ }^{4}$ State Key Laboratory of Agrobiotechnology, College of Biological Sciences, China Agricultural University, 100193 Beijing, People's Republic of China. ${ }^{5}$ National Engineering Laboratory for Animal Breeding, Key Laboratory of Animal Genetics, Breeding and Reproduction of the Ministry of Agriculture, College of Animal Science and Technology, China Agricultural University, 100193 Beijing, People's Republic of China

\section{Author contributions}

W.H., W.C., and W.S. designed research; L.J., W.W., X.Q., Z.C., W.J., and N.Z. performed research; W.H., W.C., W.S., L.J., W.W., X.Q., H.B., and K.S. analyzed the data; L.J., W.W., and W.H. wrote the paper.

\section{Conflict of interest}

The authors declare that they have no conflict of interest.

\section{Publisher's note}

Springer Nature remains neutral with regard to jurisdictional claims in published maps and institutional affiliations.

Supplementary Information accompanies this paper at (https://doi.org/ 10.1038/s41419-019-1683-1)
Received: 15 February 2019 Revised: 8 May 2019 Accepted: 23 May 2019 Published online: 05 June 2019

\section{References}

1. Rossant, J. \& Cross, J. C. Placental development: lessons from mouse mutants. Nat. Rev. Genet. 2, 538-548 (2001).

2. Watson, E. D. \& Cross, J. C. Development of structures and transport functions in the mouse placenta. Physiology 20, 180-193 (2005).

3. Maltepe, E., Bakardjiev, A. I. \& Fisher, S. J. The placenta: transcriptional, epigenetic, and physiological integration during development. J. Clin. Invest. 120 1016-1025 (2010)

4. Rai, A. \& Cross, J. C. Development of the hemochorial maternal vascular spaces in the placenta through endothelial and vasculogenic mimicry. Dev. Biol. 387, 131-141 (2014).

5. Downs, K. M. Early placental ontogeny in the mouse. Placenta 23, 116-131 (2002).

6. Cross, J. C., Simmons, D. G. \& Watson, E. D. Chorioallantoic morphogenesis and formation of the placental villous tree. Ann. N.Y. Acad. Sci. 995, 84-93 (2003).

7. Chi, L. et al. G9a controls placental vascular maturation by activating the Notch pathway. Development 144, 1976-1987 (2017).

8. Gasperowicz, M., Surmann-Schmitt, C., Hamada, Y., Otto, F. \& Cross, J. C. The transcriptional co-repressor TLE3 regulates development of trophoblast giant cells lining maternal blood spaces in the mouse placenta. Dev. Biol. 382, 1-14 (2013).

9. Coan, P. M., Conroy, N., Burton, G. J. \& Ferguson-Smith, A. C. Origin and characteristics of glycogen cells in the developing murine placenta. Dev. Dyn. 235, 3280-3294 (2006).

10. Cross, J. C. et al. Trophoblast functions, angiogenesis and remodeling of the maternal vasculature in the placenta. Mol. Cell. Endocrinol. 187, 207-212 (2002).

11. Harris, L. K. Review: Trophoblast-vascular cell interactions in early pregnancy: how to remodel a vessel. Placenta 31(Suppl), S93-S98 (2010).

12. Inman, K. E. \& Downs, K. M. The murine allantois: emerging paradigms in development of the mammalian umbilical cord and its relation to the fetus. Genesis 45, 237-258 (2007)

13. Gasperowicz, M. \& Otto, F. The notch signalling pathway in the development of the mouse placenta. Placenta 29, 651-659 (2008).

14. Haider, S., Pollheimer, J. \& Knofler, M. Notch signalling in placental development and gestational diseases. Placenta 56, 65-72 (2017).

15. Guruharsha, K. G., Kankel, M. W. \& Artavanis-Tsakonas, S. The Notch signalling system: recent insights into the complexity of a conserved pathway. Nat. Rev. Genet. 13, 654-666 (2012)

16. Furukawa, T. et al. The Drosophila RBP-J kappa gene encodes the binding protein for the immunoglobulin J kappa recombination signal sequence. J. Biol. Chem. 266, 23334-23340 (1991).

17. Oka, C. et al. Disruption of the mouse RBP-J kappa gene results in early embryonic death. Development 121, 3291-3301 (1995).

18. Zhang, S. et al. Uterine Rbpj is required for embryonic-uterine orientation and decidual remodeling via Notch pathway-independent and -dependent mechanisms. Cell Res. 24, 925-942 (2014).

19. Anson-Cartwright, L. et al. The glial cells missing-1 protein is essential for branching morphogenesis in the chorioallantoic placenta. Nat. Genet. 25, 311-314 (2000)

20. Basyuk, E. et al. Murine Gcm1 gene is expressed in a subset of placental trophoblast cells. Dev. Dyn. 214, 303-311 (1999).

21. Simmons, D. G. et al. Early patterning of the chorion leads to the trilaminar trophoblast cell structure in the placental labyrinth. Development 135, 2083-2091 (2008)

22. Hayashi, S., Lewis, P., Pevny, L. \& McMahon, A. P. Efficient gene modulation in mouse epiblast using a Sox2Cre transgenic mouse strain. Gene Expr. Patterns $\mathbf{2}$, 93-97 (2002).

23. Gurtner, G. C. et al. Targeted disruption of the murine VCAM1 gene: essential role of VCAM-1 in chorioallantoic fusion and placentation. Genes Dev. 9, 1-14 (1995).

24. Yang, J. T., Rayburn, H. \& Hynes, R. O. Cell adhesion events mediated by alpha 4 integrins are essential in placental and cardiac development. Development 121, 549-560 (1995)

25. Tun, T. et al. Recognition sequence of a highly conserved DNA binding protein RBP-J kappa. Nucleic Acids Res. 22, 965-971 (1994).

26. Limbourg, F. P. et al. Essential role of endothelial Notch1 in angiogenesis. Circulation 111, 1826-1832 (2005).

27. Tu, L. et al. Notch signaling is an important regulator of type 2 immunity. J. Exp. Med. 202, 1037-1042 (2005) 
28. Shawber, C. J., Brown-Grant, D. A., Wu, T., Kitajewski, J. K. \& Douglas, N. C. Dominant-negative inhibition of canonical Notch signaling in trophoblast cells does not disrupt placenta formation. Biol. Open 8, bio037721 (2019).

29. Riley, P., Anson-Cartwright, L. \& Cross, J. C. The Hand1 bHLH transcription factor is essential for placentation and cardiac morphogenesis. Nat. Genet. 18 271-275 (1998)

30. Guillemot, F., Nagy, A., Auerbach, A., Rossant, J. \& Joyner, A. L. Essential role of Mash-2 in extraembryonic development. Nature 371, 333-336 (1994).

31. Simmons, D. G., Fortier, A. L. \& Cross, J. C. Diverse subtypes and developmental origins of trophoblast giant cells in the mouse placenta. Dev. Biol. 304 567-578 (2007).

32. Hu, D. \& Cross, J. C. Ablation of Tpbpa-positive trophoblast precursors leads to defects in maternal spiral artery remodeling in the mouse placenta. Dev. Biol. 358, 231-239 (2011).

33. Ohsako, S., Hyer, J., Panganiban, G., Oliver, I. \& Caudy, M. Hairy function as a DNA-binding helix-loop-helix repressor of Drosophila sensory organ formation. Genes Dev. 8, 2743-2755 (1994).

34. Van Doren, M., Bailey, A. M., Esnayra, J., Ede, K. \& Posakony, J. W. Negative regulation of proneural gene activity: hairy is a direct transcriptional repressor of achaete. Genes Dev. 8, 2729-2742 (1994).

35. Calzonetti, T., Stevenson, L. \& Rossant, J. A novel regulatory region is required for trophoblast-specific transcription in transgenic mice. Dev. Biol. 171 615-626 (1995).

36. Kwee, $\mathrm{L}$. et al. Defective development of the embryonic and extraembryonic circulatory systems in vascular cell adhesion molecule (VCAM-1) deficient mice. Development 121, 489-503 (1995).

37. Krebs, L. T. et al. Haploinsufficient lethality and formation of arteriovenous malformations in Notch pathway mutants. Genes Dev. 18, 2469-2473 (2004).

38. Gasperowicz, M., Rai, A. \& Cross, J. C. Spatiotemporal expression of Notch receptors and ligands in developing mouse placenta. Gene Expr. Patterns $\mathbf{1 3}$ 249-254 (2013).

39. Gale, N. W. et al. Haploinsufficiency of delta-like 4 ligand results in embryonic lethality due to major defects in arterial and vascular development. Proc. Natl Acad. Sci. USA 101, 15949-15954 (2004).
40. Duarte, A. et al. Dosage-sensitive requirement for mouse Dll4 in artery development. Genes Dev. 18, 2474-2478 (2004).

41. Tanaka, M., Gertsenstein, M., Rossant, J. \& Nagy, A. Mash2 acts cell autonomously in mouse spongiotrophoblast development. Dev. Biol. 190, 55-65 (1997).

42. Hunkapiller, N. M. et al. A role for Notch signaling in trophoblast endovascular invasion and in the pathogenesis of pre-eclampsia. Development $\mathbf{1 3 8}$, 2987-2998 (2011)

43. Hamada, Y. et al. Notch2 is required for formation of the placental circulatory system, but not for cell-type specification in the developing mouse placenta. Differentiation 75, 268-278 (2007)

44. Tanigaki, K. \& Honjo, T. Two opposing roles of RBP-J in Notch signaling. Curr. Top. Dev. Biol. 92, 231-252 (2010).

45. Hori, K. et al. A nonclassical bHLH Rbpj transcription factor complex is required for specification of GABAergic neurons independent of Notch signaling. Genes Dev. 22, 166-178 (2008).

46. Diaz-Trelles, R. et al. Notch-independent RBPJ controls angiogenesis in the adult heart. Nat. Commun. 7, 12088 (2016).

47. Mould, A., Morgan, M. A., Li, L., Bikoff, E. K. \& Robertson, E. J. Blimp1/Prdm1 governs terminal differentiation of endovascular trophoblast giant cells and defines multipotent progenitors in the developing placenta. Genes Dev. 26 2063-2074 (2012)

48. Han, $\mathrm{H}$. et al. Inducible gene knockout of transcription factor recombination signal binding protein-J reveals its essential role in T versus B lineage decision. Int. Immunol. 14, 637-645 (2002).

49. Wenzel, P. L. \& Leone, G. Expression of Cre recombinase in early diploid trophoblast cells of the mouse placenta. Genesis 45, 129-134 (2007).

50. Das, S. K. et al. Heparin-binding EGF-like growth factor gene is induced in the mouse uterus temporally by the blastocyst solely at the site of its apposition: a possible ligand for interaction with blastocyst EGF-receptor in implantation. Development 120, 1071-1083 (1994).

51. Nagy, A., Rossant, J., Nagy, R., Abramow-Newerly, W. \& Roder, J. C. Derivation of completely cell culture-derived mice from early-passage embryonic stem cells. Proc. Natl Acad. Sci. USA 90, 8424-8428 (1993). 